\title{
Levels of urinary isoflavones and lignan polyphenols in Japanese women
}

\author{
Wanyang Liu $\cdot$ Miyako Tanabe $\cdot$ Kouji H. Harada $\cdot$ \\ Akio Koizumi
}

Received: 14 December 2012/Accepted: 29 March 2013/Published online: 30 April 2013

(C) The Japanese Society for Hygiene 2013

\begin{abstract}
Objectives High consumption of soybean products has been associated with a reduced risk of hormone-sensitive tumors. Soybean products contain phytoestrogens, such as daidzein, and sesame seeds contain secoisolariciresinol. These compounds are further metabolized to equol, enterodiol, and enterolactone by intestinal bacteria. However, individual differences in the metabolizing potential remain unclear. The aim of this study was to evaluate the urinary daidzein, equol, enterodiol, and enterolactone concentrations in women from several different regions of Japan according to age group.

Methods Five hundred urine samples collected from Japanese women living in Sapporo, Sendai, Kyoto, Kochi, and Naha were analyzed for daidzein, equol, enterodiol, and enterolactone concentration by gas chromatographymass spectrometry.

Results The urinary isoflavone and lignan polyphenol levels did not differ significantly among the sampling sites, except for daidzein, which was highest in urine collected at Naha. The prevalence of equol producers was $39 \%$ in the total study cohort. In equol producers, a positive correlation was observed between the urinary daidzein and equol levels $(r=0.399, p<0.001)$. However, there was no significant difference between daidzein concentrations in
\end{abstract}

W. Liu $\cdot$ M. Tanabe $\cdot$ K. H. Harada $\cdot$ A. Koizumi $(\square)$ Department of Health and Environmental Sciences, Kyoto University Graduate School of Medicine, Yoshida Konoe, Sakyo, Kyoto 606-8501, Japan

e-mail: Koizumi.Akio.5v@kyoto-u.ac.jp

W. Liu

Department of Occupational and Environmental Health, School of Public Health, China Medical University, Shenyang 110001, People's Republic of China equol producers and non-producers. Moreover, the levels of enterodiol and enterolactone were higher in equol producers than in equol non-producers. In the multivariate logistic analyses, two factors, Sendai dwelling and current smoking, were found to be significant [equol producers to non-producers: odds ratio 2.15 (95\% confidence interval: 1.17-4.02) and odds ratio 0.32 (0.15-0.63), respectively]. Conclusions Our data suggest that geographic factors and smoking status should be considered during the evaluation of equol in urine samples and that the same pathway may be responsible for the metabolism of both isoflavones and lignan polyphenols.

Keywords Phytoestrogen · Isoflavone - Lignan polyphenol $\cdot$ Equol producer $\cdot$ Japanese

\section{Introduction}

The risks of breast cancer and prostate cancer in Japan tend to be lower than those in Europe and the USA [1]. Differences in food habits are thought to be one of the reasons for this difference [2], which has led researchers to focus on the high consumption of soybean products by Japanese. Soybean products contain phytoestrogens with isoflavone structures, such as daidzein [3], while sesame seeds also contain lignan polyphenols, such as secoisolariciresinol. These polyphenolic compounds bind to estrogen receptors alpha and beta, with a preference for the latter [4]. Several epidemiological studies have suggested that high phytoestrogen levels are associated with a reduced risk of hormone-sensitive diseases [5-10].

These compounds are further metabolized to equol, enterodiol, and enterolactone by intestinal bacteria [11]. These metabolites, especially equol, show more potent 
binding affinities to estrogen receptors [12] than the respective substrate polyphenolic compound. However, they do show individual differences in metabolizing potential [13-15], and factors such as age, ethnicity, dietary fiber intake, and fat intake have been reported as candidate determinants for these differences. In addition, given that dietary and life habits are candidate determinants, there may be intergenerational and interzonal differences.

The aim of this study was to evaluate the concentrations of daidzein, equol, enterodiol, and enterolactone in urine samples from five regions in Japan according to broad age groups.

\section{Materials and methods}

Experimental design and study population

A total of 13,910 participants were originally recruited through medical check-ups of participants aged 20-70 years living in 11 prefectures in Japan between 2000 and 2001 [16]. Age, number of births, smoking habit, and menstrual function were recorded using a self-reported questionnaire. Urine samples were stored at $-30{ }^{\circ} \mathrm{C}$ until analysis at the Kyoto University Human Specimen Bank $[17,18]$.

To evaluate geographical differences in Japan, we compared 500 samples collected from Hokkaido (Sapporo), Miyagi (Sendai), Kyoto (Kyoto), Kochi (Kochi), and Okinawa (Naha) between November 2000 and December 2001. At each study site, urine samples were collected from 25 adult Japanese females ranging in age across four age groups $(30-39,40-49,50-59$, and $60-69$ years). The characteristics of the participants are summarized in Table 1. There were no significant differences in participant characteristics among the five study sites.

Written informed consent was obtained from all subjects prior to participation in the study. The research protocol for the study was reviewed and approved by the Ethics
Committee of Kyoto University Graduate School of Medicine on 14 November 2003 (E25). All experiments were carried out in compliance with the Helsinki Declaration.

\section{Reagents}

Daidzein, equol, enterodiol, and enterolactone were obtained from Fujicco Co. Ltd. (Kobe, Japan), Enzo Life Sciences Inc. (Farmingdale, NY), ChromaDex Inc. (Irvine, CA), and Cayman Chemical Company (Ann Arbor, MI), respectively. $\mathrm{D}_{6}$-daidzein was purchased from Toronto Research Chemicals Inc. (North York, ON, Canada). Methanol, ethyl acetate, and acetonitrile (pesticide analysis grade) were obtained from Kanto Chemicals (Tokyo, Japan). Helix pomatia-derived glucuronidase/sulfatase was purchased from Sigma-Aldrich Inc. (St. Louis, MO). Methyl t-butyl ether (pesticide analysis grade) and ascorbic acid were purchased from Wako Pure Chemicals (Osaka, Japan). N,O-bis(trimethylsilyl)trifluoroacetamide with $1 \%$ trimethylchlorosilane was obtained from Thermo Fisher Scientific Inc. (Waltham, MA).

\section{Determination of phytoestrogens in urine}

Daidzein, equol, enterodiol, and enterolactone were analyzed. The urine samples were subjected to a clean-up procedure using a solid-phase extraction. Briefly, $0.5 \mathrm{~mL}$ of a urine sample, $50 \mu \mathrm{L}$ of $0.1 \mathrm{M}$ ascorbic acid, and an internal standard (20 $\mathrm{ng} \mathrm{D}_{6}$-daidzein) were placed in a 1.5$\mathrm{mL}$ polypropylene tube and $20 \mu \mathrm{L}$ of glucuronidase/sulfatase solution $(2,500 \mathrm{U})$ was added. The samples were shaken on a vertical shaker overnight at $37{ }^{\circ} \mathrm{C}$, and then each solution was passed through a Sep-Pak Plus $\mathrm{C}_{18}$ solidphase cartridge (particle size 55-105 $\mu \mathrm{m}$; sorbent weight $360 \mathrm{mg}$; Waters Corp., Milford, MA) previously conditioned with $4 \mathrm{~mL}$ of methanol and $4 \mathrm{~mL}$ of $5 \%$ methanol in water. Subsequent loading of the sample was followed by washing the sorbent with $4 \mathrm{~mL}$ of $5 \%$ methanol in water. The analytes were eluted into a glass tube using

Table 1 Characteristics of the study population

\begin{tabular}{|c|c|c|c|c|c|c|c|}
\hline Characteristics & Total & Hokkaido & Miyagi & Kyoto & Kochi & Okinawa & $p$ value $^{\mathrm{a}}$ \\
\hline Age $($ mean $\pm \mathrm{SD})$ & $49.2 \pm 10.1$ & $49.4 \pm 10.7$ & $50.1 \pm 10.7$ & $48.9 \pm 9.4$ & $49.3 \pm 10.1$ & $48.5 \pm 9.9$ & 0.8 \\
\hline Number of delivery (mean \pm SD) & $1.9 \pm 1$ & $1.7 \pm 0.9$ & $2.0 \pm 0.9$ & $1.8 \pm 0.9$ & $1.9 \pm 0.9$ & $2.0 \pm 1.4$ & 0.3 \\
\hline Post menopause $(\%)$ & 37 & 34 & 33 & 46 & 36 & 37 & 0.6 \\
\hline \multicolumn{8}{|l|}{ Smoking habit $(\%)$} \\
\hline Non-smoker & 86 & 83 & 83 & 91 & 80 & 90 & 0.2 \\
\hline Current smoker & 11 & 13 & 15 & 8 & 15 & 7 & \\
\hline Ex-smoker & 3 & 4 & 2 & 1 & 5 & 3 & \\
\hline
\end{tabular}

SD Standard deviation

${ }^{\text {a }}$ Differences among residential areas were tested by analysis of variance or the $\chi^{2}$ test 
$3 \mathrm{~mL}$ of $1: 1(\mathrm{v} / \mathrm{v})$ acetonitrile and ethyl acetate. The solution was evaporated to $1 \mathrm{~mL}$ using dry $\mathrm{N}_{2}$ and extracted with $2 \mathrm{~mL}$ of methyl t-butyl ether. The methyl t-butyl ether layer was dried up using dry $\mathrm{N}_{2}$, after which $100 \mu \mathrm{L}$ of methyl t-butyl ether and $50 \mu \mathrm{L}$ of $N, O$-bis(trimethylsilyl)trifluoroacetamide with $1 \%$ trimethylchlorosilane were added. The solution was transferred to an autosampler vial and heated for $1 \mathrm{~h}$ at $60{ }^{\circ} \mathrm{C}$. The extracts were analyzed by gas chromatography-mass spectrometry (model 6890GC/5973MSD; Agilent Technologies Japan Ltd., Tokyo, Japan) in the electron impact ionization mode using single ion monitoring. The trimethylsilyl derivatives were separated on a DB-5MS column (length $30 \mathrm{~m}$, inner diameter $0.25 \mathrm{~mm}$, film thickness $0.25 \mu \mathrm{m}$ ) with a helium carrier gas. Splitless injections $(1 \mu \mathrm{L})$ were performed with the injector set at $280{ }^{\circ} \mathrm{C}$, and the split was opened after $1.5 \mathrm{~min}$. The oven temperature was initially $200{ }^{\circ} \mathrm{C}$, then ramped to $300{ }^{\circ} \mathrm{C}$ at $30^{\circ} \mathrm{C} / \mathrm{min}$, and held for $12 \mathrm{~min}$. The monitored ions are listed in Electronic Supplementary Material Table 1. The instrumental detection limits (IDLs) were defined as the mass of analyte producing a peak with a signal-to-noise ratio of 3 , and ranged from 2 (enterodiol) to $100 \mathrm{pg}$ (daidzein) (Table 2). Since blank samples $(0.5 \mathrm{~mL}$ of distilled water) contained no detectable concentrations, the method detection limits (MDLs) were considered to be equal to the IDLs, corresponding to $0.6 \mathrm{ng} / \mathrm{mL}$ for enterodiol and $28 \mathrm{ng} / \mathrm{mL}$ for daidzein (Table 2).

\section{Quality assurance}

Quantification was performed using an internal standard method with the external standards dissolved in $100 \mu \mathrm{L}$ of methyl t-butyl ether and $50 \mu \mathrm{L}$ of $\mathrm{N}, \mathrm{O}$-bis(trimethylsilyl)trifluoroacetamide with $1 \%$ trimethylchlorosilane. $\mathrm{D}_{6}$-labeled daidzein was used as the internal standard for all analytes. All samples were quantified using a sevenpoint calibration curve, with a relative standard deviation (RSD) of the relative response factors of $<15 \%$ for all compounds. The recoveries were evaluated by ten replicate fortifications (fortified by $10 \times$ the original concentration of urine) of a sample from equol non-producers (Table 2). The procedural blank levels were evaluated in duplicate for 11 samples each using $0.5 \mathrm{~mL}$ of distilled water.

\section{Statistical analysis}

All statistical analyses were carried out using JMP software (ver. 4; SAS Institute, Cary, NC). Values of $p<0.05$ were considered to indicate statistical significance. Concentrations of less than the detection limit were all approximated to half of the detection limit for statistical analyses. The urinary levels of phytoestrogens were corrected by the urinary creatinine $(\mathrm{Cr})$ concentration. As the levels in the samples displayed right-skewed patterns, and the geometric means (GMs) were comparable to the medians, statistical analyses were conducted after log-transformation of the concentrations. Differences between mean values were tested by Tukey-Kramer's honestly significant difference test after analysis of variance or Student's $t$ test. To reveal the relationships between equol-producing function and subjects' characteristics, we performed multivariate logistic analyses. Presence of equol-producing function was defined by the detection of equol in urine samples above the MDL.

\section{Results}

Urinary levels of phytoestrogens in study cohort of Japanese women

The descriptive statistics for the phytoestrogens levels are presented in Table 3. All samples contained detectable amounts of daidzein and enterolactone. Equol and enterodiol were detected in 39 and $77 \%$ of samples, respectively. The GMs were as follows: daidzein, $1610 \mu \mathrm{g} / \mathrm{g}-\mathrm{Cr}$; equol, $78.4 \mu \mathrm{g} / \mathrm{g}-\mathrm{Cr}$; enterolactone, $36.7 \mu \mathrm{g} / \mathrm{g}-\mathrm{Cr}$; enterodiol,

Table 2 Recovery, detection limits, and quality assurance for isoflavones and lignan polyphenols in human urine samples

\begin{tabular}{|c|c|c|c|c|}
\hline Compound & $\begin{array}{l}\text { Quantification } \\
\text { (confirmation value) }\end{array}$ & $\begin{array}{l}\text { Recovery }(\%) \text { and } \\
\text { (reproducibility, RSD \%) } \\
(n=10)\end{array}$ & $\begin{array}{l}\text { Instrument detection } \\
\text { limit }^{\mathrm{a}}(\mathrm{pg})\end{array}$ & $\begin{array}{l}\text { Method detection } \\
\text { limit }^{\mathrm{b}}(\mathrm{ng} / \mathrm{mL})\end{array}$ \\
\hline Equol & $386(371)$ & $103.2(9.4)$ & 60 & 20 \\
\hline Daidzein & 398 (383) & $98.9(7.3)$ & 100 & 28 \\
\hline Enterodiol & $410(500)$ & $94.7(9.2)$ & 2 & 0.6 \\
\hline Enterolactone & $442(263)$ & $97.3(10.0)$ & 10 & 3 \\
\hline $\mathrm{D}_{6}$-daidzein & 404 (389) & - & - & - \\
\hline
\end{tabular}

$R S D$ Relative standard deviation

a $1-\mu$ injection

b 0.5 -mL urine sample 
Table 3 Geometric means, medians, and 95th percentiles of urinary phytoestrogen concentrations in our study cohort of Japanese women

\begin{tabular}{|c|c|c|c|c|}
\hline \multirow[t]{2}{*}{ Sample sites } & \multicolumn{2}{|c|}{ Isoflavones (soybean) ${ }^{\mathrm{a}}$} & \multicolumn{2}{|c|}{ Lignan polyphenols (sesame seeds) ${ }^{\mathrm{a}}$} \\
\hline & Equol ( $\mu \mathrm{g} / \mathrm{g}-\mathrm{Cr})$ & Daidzein $(\mu \mathrm{g} / \mathrm{g}-\mathrm{Cr})$ & Enterolactone $(\mu \mathrm{g} / \mathrm{g}-\mathrm{Cr})$ & Enterodiol $(\mu \mathrm{g} / \mathrm{g}-\mathrm{Cr})$ \\
\hline \multirow[t]{3}{*}{ Total } & $78.4(6.4)$ & $1,610(4.6)$ & $36.7(6.0)$ & $23.8(7.1)$ \\
\hline & - & 1,946 & 42.3 & 28.8 \\
\hline & 1,385 & 6,761 & 279 & 228 \\
\hline \multirow[t]{3}{*}{ Hokkaido } & $56.8(6.4)$ & $1,509(5.2)^{\mathrm{a}, \mathrm{b}}$ & 34.7 (6.2) & $19.2(7.1)$ \\
\hline & - & 1,758 & 45.9 & 27.4 \\
\hline & 1,325 & 7,120 & 248 & 166 \\
\hline \multirow[t]{3}{*}{ Miyagi } & $110.6(6.8)$ & $1,454(4.7)^{\mathrm{a}}$ & $28.7(6.0)$ & $20.6(7.0)$ \\
\hline & - & 1,785 & 35.4 & 24.3 \\
\hline & 1,827 & 7,120 & 229 & 179 \\
\hline \multirow[t]{3}{*}{ Kyoto } & $75.2(6.8)$ & $1,334(4.7)^{\mathrm{a}}$ & $48.6(6.1)$ & $36.8(7.2)$ \\
\hline & - & 1,541 & 50.1 & 61.3 \\
\hline & 1,285 & 6,380 & 350 & 310 \\
\hline \multirow[t]{3}{*}{ Kochi } & $66.5(6.1)$ & $1,419(5.5)^{\mathrm{a}}$ & $41.6(6.0)$ & $25.0(7.2)$ \\
\hline & - & 1,634 & 50.1 & 30.3 \\
\hline & 1,382 & 9,063 & 295 & 240 \\
\hline \multirow[t]{3}{*}{ Okinawa } & $93.2(5.9)$ & $2,616(2.6)^{b}$ & $32.7(5.8)$ & $20.3(6.8)$ \\
\hline & - & 2,762 & 38.3 & 24.2 \\
\hline & 812 & 5,025 & 203 & 200 \\
\hline
\end{tabular}

GM Geometric mean, GSD geometric standard deviation

Comparisons were made among the residential areas. The geometric means within the same column followed by the same lower-case letter are significantly different $(p<0.05)$. The geometric means within the same column followed by the same lower-case letter of not followed by a lower-case letter do not differ significantly $(p>0.05)$

${ }^{a}$ Urinary phytoestrogen concentrations for the total study cohort and for each study region are given as: GM (GSD) (top row), median (middle row), and 95th percentile (bottom row)

$23.8 \mu \mathrm{g} / \mathrm{g}-\mathrm{Cr}$. The $95 \mathrm{th}$ percentiles were as follows: daidzein, $6761 \mu \mathrm{g} / \mathrm{g}-\mathrm{Cr}$; equol, $1385 \mu \mathrm{g} / \mathrm{g}-\mathrm{Cr}$; enterolactone, $279 \mu \mathrm{g} / \mathrm{g}-\mathrm{Cr}$; enterodiol, $228 \mu \mathrm{g} / \mathrm{g}$-Cr. The urinary daidzein level was highest in Okinawa, followed by Hokkaido and the other sites $(p<0.05)$. There were no significant differences between the GMs for equol, enterodiol, and enterolactone $(p>0.05)$.

Urinary phytoestrogen concentrations were also compared between the equol producers and non-producers (Table 4). In both groups, no significant differences in the GMs of the phytoestrogens were observed among the five sampling sites $(p>0.05)$. The urinary levels of daidzein were comparable between the equol producers and nonproducers $(p>0.05)$. In contrast, the GMs of enterodiol and enterolactone were higher in the equol producers than in the non-producers $(p<0.001)$. These trends were consistent over all five sampling sites.

The correlation coefficients among the phytoestrogens in the 500 samples are listed in Table 5. Significant correlations were observed between enterolactone and enterodiol in both the equol non-producers and producers $(\rho=0.592$ and $\rho=0.622$, respectively). In the equol producers, the equol concentration was significantly associated with not only the daidzein concentration ( $\rho=0.399)$ but also the enterolactone and enterodiol concentrations ( $\rho=0.162$ and $\rho=0.149$, respectively).

Relationships between equol-metabolizing function and participants' characteristics

The demographic status of equol producers and non-producers is summarized in Table 6. There were no significant differences in age, number of births, and ratio of postmenopause $(p>0.05)$. The smoker ratio was 6 and $15 \%$ in the equol producers and non-producers, respectively, which was a significant difference $(p=0.009)$. The samples from Miyagi showed a marginally higher ratio of equol producers than those from the other four sites $(p=0.16)$. In multivariate logistic analyses, two factors, Miyagi residence and current smoking, were significantly associated with equol-producing function [equol producers to non-producers: odds ratio 2.15 , (95\% confidence interval 1.17-4.02) and odds ratio 0.32 (0.15-0.63), respectively]. 
Table 4 Urinary phytoestrogen concentrations in equol producers and non-producers

\begin{tabular}{|c|c|c|c|c|}
\hline \multirow[t]{2}{*}{ Equol producers and non-producers } & \multicolumn{2}{|c|}{ Isoflavones (soybean) ${ }^{\mathrm{a}}$} & \multicolumn{2}{|c|}{ Lignan polyphenols (sesame seeds) ${ }^{\mathrm{a}}$} \\
\hline & Equol $(\mu \mathrm{g} / \mathrm{g}-\mathrm{Cr})$ & Daidzein $(\mu \mathrm{g} / \mathrm{g}-\mathrm{Cr})$ & Enterolactone $(\mu \mathrm{g} / \mathrm{g}-\mathrm{Cr})$ & Enterodiol $(\mu \mathrm{g} / \mathrm{g}-\mathrm{Cr})$ \\
\hline \multicolumn{5}{|l|}{ Total } \\
\hline $\mathrm{E}(+)$ & $564(4.0)$ & $1,531(4.6)$ & $56.7(4.4)^{* * *}$ & $62.4(4.4) * * *$ \\
\hline$(n=195)$ & 565 & 1,825 & 63.1 & 74.8 \\
\hline $\mathrm{E}(-)$ & - & $1,662(4.5)$ & $27.8(6.9)$ & $12.8(7.3)$ \\
\hline$(n=305)$ & - & 2,010 & 30.6 & 10.1 \\
\hline \multicolumn{5}{|l|}{ Hokkaido } \\
\hline $\mathrm{E}(+)$ & $681(3.7)$ & $1,738(4.8)$ & $57.3(4.4)$ & $48.3(3.7)^{* * *}$ \\
\hline$(n=30)$ & 916 & 1,779 & 42.7 & 58.6 \\
\hline $\mathrm{E}(-)$ & - & $1,420(5.4)$ & $27.9(6.8)$ & $13.0(7.9)$ \\
\hline$(n=70)$ & - & 1,758 & 46.3 & 11.54 \\
\hline \multicolumn{5}{|l|}{ Miyagi } \\
\hline $\mathrm{E}(+)$ & $606(3.9)$ & $1,191(4.8)$ & $35.7(4.8)$ & $54.4(4.1)^{* * *}$ \\
\hline$(n=47)$ & 636 & 1,324 & 41.8 & 70.8 \\
\hline $\mathrm{E}(-)$ & - & $1,736(4.5)$ & $23.7(7.2)$ & $8.6(7.1)$ \\
\hline$(n=53)$ & - & 2,292 & 27.7 & 5.8 \\
\hline \multicolumn{5}{|l|}{ Kyoto } \\
\hline $\mathrm{E}(+)$ & $614(4.3)$ & $1,164(4.1)$ & $70.9(4.0)$ & $101.7(3.4)^{* * *}$ \\
\hline$(n=38)$ & 518 & 1,265 & 68.1 & 88.8 \\
\hline $\mathrm{E}(-)$ & - & $1,448(5.0)$ & 38.7 (7.5) & $19.9(8.1)$ \\
\hline$(n=62)$ & - & 1,976 & 38.7 & 17.7 \\
\hline \multicolumn{5}{|l|}{ Kochi } \\
\hline $\mathrm{E}(+)$ & 404 (4.7) & $1,361(6.0)$ & $78.1(3.7)^{* *}$ & $62.5(4.8)^{* * *}$ \\
\hline$(n=40)$ & 382 & 1,647 & 78.6 & 61.1 \\
\hline $\mathrm{E}(-)$ & - & $1,459(5.4)$ & $27.4(6.9)$ & $13.7(7.3)$ \\
\hline$(n=60)$ & - & 1,536 & 22.4 & 9.2 \\
\hline \multicolumn{5}{|l|}{ Okinawa } \\
\hline $\mathrm{E}(+)$ & $579(3.5)$ & $2,745(3.1)$ & $56.6(4.6)^{* *}$ & $54.4(5.6)^{* * *}$ \\
\hline$(n=40)$ & 490 & 3,520 & 70.3 & 64.6 \\
\hline $\mathrm{E}(-)$ & - & $2,533(2.3)$ & $22.5(6.1)$ & $10.4(5.9)$ \\
\hline$(n=60)$ & - & 2,570 & 24.5 & 6.0 \\
\hline
\end{tabular}

** $p<0.01, * * * p<0.001$, vs. equol non-producers by Student's $t$ test after log-transformation

$E(+)$ Equol producers, $E(-)$ equol non-producers, $\mathrm{Cr}$ creatinine

${ }^{a}$ Urinary phytoestrogen concentrations for equol producers and non-producers in the total study cohort and for each study region are given as: GM (GSD) (top row) and median (bottom row)

\section{Discussion}

We have evaluated the urinary levels of phytoestrogens in a sample of women residing in five different areas of Japan. Daidzein was found to be the predominant component of the phytoestrogens present in the urine of the Japanese women sampled and lignan polyphenols were minor components.

Overall, $39 \%$ of the urine samples contained detectable levels of equol. Equol-producing function was also associated with smoking status. Previous studies in Japan reported equol detection rates of $20 \%$ in female subjects
[15] and $24 \%$ in male subjects [19]. In our study, the equol detection rates ranged from 30 to $47 \%$. These variations might be associated with differences in dietary and life habits. Indeed, we found an association between smoking and low equol-producing function. A previous study indicated that the proportion of equol producers is low in young males compared with older males [19]. In our study, no such trend was observed in the study women. This trend is likely to reflect the smoking rate in each age group in Japan [20]. Although the proportion of daidzein to equol in the urine was around $30 \%$ in equol producers, there was no significant difference in the urinary levels of daidzein 
Table 5 Correlations among phytoestrogens

\begin{tabular}{lrr}
\hline Combination & $\rho$ & $p$ value \\
\hline Equol non-producers & & \\
Enterodiol-Daidzein & -0.057 & 0.309 \\
Enterolactone-Daidzein & -0.003 & 0.959 \\
Enterolactone-Enterodiol & 0.592 & $<0.001$ \\
Equol producers & & \\
Daidzein-Equol & 0.399 & $<0.001$ \\
Enterodiol-Equol & 0.149 & 0.034 \\
Enterodiol-Daidzein & -0.059 & 0.403 \\
Enterolactone-Equol & 0.162 & 0.021 \\
Enterolactone-Daidzein & -0.123 & 0.079 \\
Enterolactone-Enterodiol & 0.622 & $<0.001$ \\
\hline
\end{tabular}

$\rho$ Spearman's rank correlation coefficient

Table 6 Relationships between equol-producing status and demographic characteristics

\begin{tabular}{llll}
\hline $\begin{array}{l}\text { Demographic } \\
\text { characteristics }\end{array}$ & $\begin{array}{l}\text { Equol } \\
\text { producer }\end{array}$ & $\begin{array}{l}\text { Equol non- } \\
\text { producer }\end{array}$ & $\begin{array}{l}\text { Odds ratio (95 \% } \\
\text { confidence interval) }\end{array}$ \\
\hline Age $^{\mathrm{b}}$ & $49.7 \pm 9.7$ & $48.9 \pm 10.4$ & $0.40(0.07-2.04)$ \\
Number of births $^{\mathrm{b}}$ & $1.9 \pm 1.0$ & $1.9 \pm 1.1$ & $0.94(0.37-2.43)$ \\
$\begin{array}{l}\text { Post-menopause } \\
(\%)\end{array}$ & 38 & 36 & $1.61(0.87-3.01)$ \\
$\begin{array}{l}\text { Smoking habit } \\
(\%)\end{array}$ & & & \\
Non-smoker & 91 & 82 & - $^{\mathrm{c}}$ \\
Current smoker & 6 & 15 & $0.32(0.15-0.63)^{*}$ \\
Ex-smoker & 3 & 3 & $0.93(0.30-2.70)$ \\
Log ${ }_{10}$ urinary & $3.2 \pm 0.7$ & $3.2 \pm 0.7$ & $0.75(0.25-2.30)$ \\
daidzein & & & \\
$\quad(\mu \mathrm{g} / \mathrm{g}-\mathrm{Cr})^{\mathrm{b}}$ & & & \\
Sampling sites $(n)$ & & & \\
Hokkaido & 30 & 70 & $-^{\mathrm{c}}$ \\
Miyagi & 47 & 53 & $2.15(1.17-4.02)^{*}$ \\
Kyoto & 38 & 62 & $1.22(0.67-2.23)$ \\
Kochi & 40 & 60 & $1.36(0.74-2.50)$ \\
Okinawa & 40 & 60 & $1.28(0.69-2.38)$ \\
\hline
\end{tabular}

* Significantly difference at $p<0.05$

a Odds ratios were calculated by multivariate logistic analyses

${ }^{\mathrm{b}}$ Continuous values are presented as the mean $\pm \mathrm{SD}$

${ }^{c}$ Set to the reference level

between equol producers and non-producers. This phenomenon could result from differences in the dietary intake of isoflavones between equol producers and non-producers or in the pharmacokinetics between equol and daidzein. Levels of another soybean isoflavone, genistein, were comparable between equol producers and non-producers (1,192 and 1,070 $\mu \mathrm{g} / \mathrm{g}-\mathrm{Cr}$, respectively). Genistein has a similar biological half-life to daidzein while the formation of 4-hydroxy-equol is considered to be rare. Therefore, dietary intake of isoflavones was unlikely to differ between two groups. The half-life of daidzein is relatively shorter than that of equol, and the conversion of daidzein into equol is time-dependent and slow [21]. Therefore, daidzein could be excreted rapidly in urine before its conversion into equol. Another possibility is that more daidzein is likely to be converted into $O$-desmethyl-angolensin (O-DMA) in non-equol producers than in equol producers [22]. This hypothesis needs to be investigated in the future.

The presence or absence of equol-producing function was clearly dichotomized despite the high daidzein levels. Intestinal microflora play important roles in the metabolism of nutrients, and the composition of the intestinal flora shows individual differences [23]. The effects of smoking on the intestinal microflora remain unknown, but smoking has been reported to influence colonic mucus production and mucosal immune systems [24]. These differences in the intestinal environment might discriminate the equolmetabolizing bacteria. As shown in Table 5, the reported association between lignan polyphenols and equol suggests that they are metabolized by the same pathway [25]. It has been suggested that some of the bacterium strains, for example, Eggerthella sp, were associated with both the metabolism of daidzein to equol and that of lignan polyphenols to enterodiol and enterolactone [26, 27]. However, the specific intestinal bacteria and pathway responsible for metabolism of these two groups of phytoestrogens need to be identified in the future. Importantly, this phenotype provides an insight for the identification of equol-metabolizing bacteria.

Acknowledgments This study was mainly supported by Special Coordination Funds for Promoting Science and Technology (No. 1300001) sponsored by the Japan Science and Technology Agency, and a Grant-in-Aid for Health Sciences Research from the Ministry of Health, Labour and Welfare of Japan (H21-Food-003).

Conflict of interest None.

\section{References}

1. Parkin DM, Whelan SL, Ferlay J, Teppo L, Thomas DB. Cancer incidence in five continents, vol. 8. IARC Scientific Publications no, 155; 2002; Geneva.

2. Messina M, Gardner C, Barnes S. Gaining insight into the health effects of soy but a long way still to go: commentary on the fourth international symposium on the role of soy in preventing and treating chronic disease. J Nutr. 2002;132[Suppl]:547-51.

3. Cassidy A, Hanley B, Lamuela-Raventos RM. Isoflavones, lignans and stilbenes: origins, metabolism and potential importance to human health. J Sci Food Agric. 2000;80:1044-62.

4. Harris DM, Besselink E, Henning SM, Go VL, Heber D. Phytoestrogens induce differential estrogen receptor alpha- or 
Beta-mediated responses in transfected breast cancer cells. Exp Biol Med (Maywood). 2005;230:558-68.

5. Iwasaki M, Inoue M, Otani T, Sasazuki S, Kurahashi N, Miura T, et al. Plasma isoflavone level and subsequent risk of breast cancer among Japanese women: a nested case-control study from the Japan Public Health Center-based prospective study group. J Clin Oncol. 2008;26:1677-83.

6. Kurahashi N, Iwasaki M, Sasazuki S, Otani T, Inoue M, Tsugane S. Soy product and isoflavone consumption in relation to prostate cancer in Japanese men. Cancer Epidemiol Biomarkers Prev. 2007;16:538-45.

7. Kurahashi N, Iwasaki M, Inoue M, Sasazuki S, Tsugane S. Plasma isoflavones and subsequent risk of prostate cancer in a nested case-control study: the Japan public health center. J Clin Oncol. 2008;26:5923-9.

8. Nagata Y, Sonoda T, Mori M, Miyanaga N, Okumura K, Goto K, et al. Dietary isoflavones may protect against prostate cancer in Japanese men. J Nutr. 2007;137:1974-9.

9. Ozasa K, Nakao M, Watanabe Y, Hayashi K, Miki T, Mikami K, et al. Association of serum phytoestrogen concentration and dietary habits in a sample set of the JACC study. J Epidemiol. 2005;15[Suppl 2]:196-202.

10. Tsuchiya M, Miura T, Hanaoka T, Iwasaki M, Sasaki H, Tanaka $\mathrm{T}$, et al. Effect of soy isoflavones on endometriosis: interaction with estrogen receptor 2 gene polymorphism. Epidemiology. 2007;18:402-8.

11. Decroos K, Vanhemmens S, Cattoir S, Boon N, Verstraete W. Isolation and characterisation of an equol-producing mixed microbial culture from a human faecal sample and its activity under gastrointestinal conditions. Arch Microbiol. 2005;183:45-55.

12. Kostelac D, Rechkemmer G, Briviba K. Phytoestrogens modulate binding response of estrogen receptors alpha and beta to the estrogen response element. J Agric Food Chem. 2003;51:7632-5.

13. Lampe JW, Karr SC, Hutchins AM, Slavin JL. Urinary equol excretion with a soy challenge: influence of habitual diet. Proc Soc Exp Biol Med. 1998;217:335-9.

14. Ozasa K, Nakao M, Watanabe Y, Hayashi K, Miki T, Mikami K, et al. Serum phytoestrogens and prostate cancer risk in a nested casecontrol study among Japanese men. Cancer Sci. 2004;95:65-71.

15. Nagata $C$, Ueno T, Uchiyama S, Nagao Y, Yamamoto S, Shibuya $\mathrm{C}$, et al. Dietary and lifestyle correlates of urinary excretion status of equol in Japanese women. Nutr Cancer. 2008;60:49-54.

16. Ezaki T, Tsukahara T, Moriguchi J, Furuki K, Fukui Y, Ukai H, et al. No clear-cut evidence for cadmium-induced renal tubular dysfunction among over 10,000 women in the Japanese general population: a nationwide large-scale survey. Int Arch Occup Environ Health. 2003;76:186-96.

17. Koizumi A, Yoshinaga T, Harada K, Inoue K, Morikawa A, Muroi J, et al. Assessment of human exposure to polychlorinated biphenyls and polybrominated diphenyl ethers in Japan using archived samples from the early 1980s and mid-1990s. Environ Res. 2005;99:31-9.

18. Koizumi A, Harada K, Inoue K, Hitomi T, Yang H-R, Moon C-S, et al. Past, present, and future of environmental specimen banks. Environ Health Prev Med. 2009;14:307-18.

19. Fujimoto K, Tanaka M, Hirao Y, Nagata Y, Mori M, Miyanaga $\mathrm{N}$, et al. Age-stratified serum levels of isoflavones and proportion of equol producers in Japanese and Korean healthy men. Prostate Cancer Prostatic Dis. 2008;11:252-7.

20. The Japanese Ministry of Health, Labour and Welfare. Smoking rate surveillance. National health and nutrition survey 2011:58. Available at: http://www.mhlw.go.jp/bunya/kenkou/eiyou/h20houkoku.html.

21. Setchell KD, Faughnan MS, Avades T, Zimmer-Nechemias L, Brown NM, Wolfe BE, et al. Comparing the pharmacokinetics of daidzein and genistein with the use of 13C-labeled tracers in premenopausal women. Am J Clin Nutr. 2003;77:411-9.

22. Possemiers S, Bolca S, Eeckhaut E, Depypere H, Verstraete W. Metabolism of isoflavones, lignans and prenylflavonoids by intestinal bacteria: producer phenotyping and relation with intestinal community. FEMS Microbiol Ecol. 2007;61:372-83.

23. Hayashi H, Sakamoto M, Benno Y. Phylogenetic analysis of the human gut microbiota using $16 \mathrm{~S}$ rDNA clone libraries and strictly anaerobic culture-based methods. Microbiol Immunol. 2002;46: 535-48.

24. Cope GF, Heatley RV. Cigarette smoking and intestinal defences. Gut. 1992;33:721-3.

25. Ishiwata N, Melby MK, Mizuno S, Watanabe S. New equol supplement for relieving menopausal symptoms: randomized, placebo-controlled trial of Japanese women. Menopause. 2009; 16:141-8.

26. Yokoyama S, Suzuki T. Isolation and characterization of a novel equol-producing bacterium from human feces. Biosci Biotechnol Biochem. 2008;72:2660-6.

27. Jin JS, Zhao YF, Nakamura N, Akao T, Kakiuchi N, Min BS, et al. Enantioselective dehydroxylation of enterodiol and enterolactone precursors by human intestinal bacteria. Biol Pharm Bull. 2007;30:2113-9. 